\title{
Recurrence and algorithmic information
}

\author{
Claudio Bonanno*, Stefano Galatolo ${ }^{\dagger}$ and Stefano Isola
}

\begin{abstract}
In this paper we initiate a somewhat detailed investigation of the relationships between quantitative recurrence indicators and algorithmic complexity of orbits in weakly chaotic dynamical systems. We mainly focus on examples.
\end{abstract}

\section{Introduction}

The general understanding of dynamical systems has recently been enriched by two important items: the quantitative characterization of recurrence from a statistical point of view and the measure of orbit complexity from an algorithmic information theoretic point of view.

The basic result about recurrence is the Poincarè Recurrence Theorem, which says that for a probability preserving transformation $(X, T, \mu)$ almost all orbits starting from a set of positive measure will come back to it infinitely many times (see, e.g., Pe] . If moreover $X$ is endowed with a metric $d$ then under suitable assumptions the sequence of distances $d_{n}=\min _{0<i \leq n} d\left(T^{i} x, x\right)$ converges to 0 as $n$ increases $[\mathrm{Fu}]$. This a qualitative statement. But what is the "typical" speed of decreasing of $d_{n}$ ? Boshernitzan started to investigate this problem in [Bo]. His results establish some bounds on the rate of decreasing of $d_{n}$ to 0 in terms of the Hausdorff dimension of the invariant measure. At the same time Ornstein and Weiss proved a probabilistic formula relating the growth of the repetition time of words of increasing length in an ergodic source with the entropy of the source [OW]. More recently, new results have been obtained by several authors relating various quantitative recurrence indicators to entropies and dimensions (see, e.g., [ACS, [BS], [STV]). On the one hand, one can measure the speed of decreasing of $d_{n}$ or else the increasing of the time $\tau_{r}$ that is necessary to come back to a neighborhood of the starting point as the radius $r$ of the neighbourhood decreases. Under suitable hyperbolicity assumptions this kind of indicators are naturally related to the dimension [BS]. On the other hand, one may

\footnotetext{
*Dipartimento di Matematica, Università di Pisa, via Buonarroti, 56126 Pisa, Italy. e-mail: $<$ bonanno@mail.dm.unipi.it>

†Dipartimento di Matematica Applicata "U. Dini", Università di Pisa, via Bonanno 25/b, 56126 Pisa, Italy. e-mail: < galatolo@mail.dm.unipi.it>

${ }_{\ddagger}^{\ddagger}$ Dipartimento di Matematica e Informatica, Università di Camerino and INFM, via Madonna delle Carceri, 62032 Camerino, Italy. e-mail: <stefano.isola@unicam.it>.
} 
study the recurrence of a given orbit with respect to the refinements of a generating partition. Under similar hypotheses this is related to the entropy of the system (see STV] and references therein).

Albeit the existing literaure is mainly devoted to the investigation of systems with positive entropy, here we study recurrence properties of some ergodic dynamical systems with zero entropy.

The entropy of a system can be interpreted as the average information that is necessary to describe one step of the evolution of the system, the underlying notion of information content being that due to Shannon. Another notion of information content is the Algorithmic Information Content (AIC) due to Kolmogorov and Chaitin. When used to investigate a dynamical system, the main difference comes from the fact that the AIC is a pointwise notion. Each digital string has its own information content, independently form the context where the string appears. Therefore the corresponding amount of information that is necessary to describe an orbit yields a pointwise version of the entropy, called orbit complexity in $[\mathrm{Br}$. Whenever a system has an invariant ergodic probability measure, the orbit complexity equals the entropy almost everywhere, hence the two notions are quantitatively related. More precisely, if the system has positive entropy $h>0$, the algorithmic information required to describe $n$ steps of an orbit increases as $h n+o(n)$ for almost every initial point.

Conversely, in a null entropy system, the (sublinear) asymptotic behaviour of the algorithmic information describing an orbit can be related to some dimensions and initial condition sensitivity indicators of the system [Ga. This suggests that the asymptotic behaviour of the algorithmic information provides a meaningful indicator to characterize the "weak" chaos present in the system.

In the following we will see how this notion is related to recurrence. More precisely we will see that low pointwise quantitative recurrence rates implies strong limitations to the increase of the information content.

The paper is organized as follows: in Section 2 we first recall several notions of recurrence which have been recently investigated in the literature and also study an indicator which measures the recurrence rate of an orbit starting from a point $x$ to a neighbourhood of some given target point $y$ (see Theorem 2.1). This will be used in the last Section to give a precise estimation of the information content for orbits generated by an Interval Exchange Transformation. We then prove some general results relating local recurrence rates to the asymptotic behaviour of the AIC.

In a sense these results are analogous to those contained in [STV], but they are meaningful for null entropy systems. These results will be used in Section 3 to estimate the local recurrence rate (for Lebesgue almost each point) for a non-uniformly hyperbolic system with an infinite invariant measure, for which the standard techniques cannot be applied.

In Section 4 we study the behaviour of the recurrence indicators introduced in the first Section along with that of the AIC for ergodic rotations and Interval Exchange Transformations. In particular in Section 4.1 we give a precise characterization of the recurrence rates for ergodic rotations in terms of arithmetic properties of the angle of rotation (thus not dimension nor entropy). From these characterizations it follows that 
the converse implication "if the information rate is low then the recurrence rate is low" does not hold.

In the last two Sections we study the behaviour of the AIC for piecewise isometries using two different approaches. In Section 4.3 by direct estimation of the algorithmic information contained in the continued fraction expansion both of the initial point and of the local rotation angle. In Section 4.4 by using the recurrence rate near given target points mentioned above. This yields sharp estimation of the information content of the orbits for a wide class of piecewise isometries of the interval.

\section{Preliminaries and some general results}

\subsection{Various kind of recurrence}

Let us first recall some general facts about recurrence. Let $(X, d)$ be a complete, separable metric space and $T: X \rightarrow X$ a Borel measurable transformation. We shall say that a point $x \in X$ is positively recurrent if

$$
\varliminf_{n \rightarrow \infty} d\left(x, T^{n} x\right)=0 .
$$

If $T$ is a homeomorphism the existence of such a point is ensured by the Birkhoff Recurrence Theorem (see $[\mathrm{Pe}]$ ). More precise results can be obtained for a metric measure preserving system (m.m.p.s) $(X, \mathcal{B}, \mu, d, T)$ where the metric $d$ on $X$ is such that open subsets of $X$ are measurable and $\mu$ is a $T$-invariant probability measure. A theorem of Furstenberg $([\mathrm{Fu}$, p.61) asserts that for a m.m.p.s. such that $(X, d)$ has a countable basis $\mu$-almost every point $x \in X$ satisfies (2.1). Quantitatively more informative result have been obtained by Boshernitzan, Barreira and Saussol involving various notions of dimension. For every $\alpha$, denote by $m_{\alpha}(\cdot)$ the Hausdorff $\alpha$-dimensional outer measure on $X$ (see e.g. [Fa]). It is well known that for any $E \subseteq X$ there is a unique value, $\operatorname{dim}_{H} E$, called the Hausdorff dimension of $E$, such that $m_{\alpha}(E)=\infty$ if $0 \leq \alpha<\operatorname{dim}_{H} E$ and $m_{\alpha}(E)=0$ if $\operatorname{dim}_{H} E<\alpha<\infty$. The Hausdorff dimension of a probability measure $\mu$ on $X$ is then given by

$$
\operatorname{dim}_{H} \mu=\inf \left\{\operatorname{dim}_{H} E: \mu(E)=1\right\} .
$$

Furthermore, the lower and upper pointwise dimensions of $\mu$ at a point $x \in X$ are given by

$$
\underline{d}_{\mu}(x)=\varliminf_{r \rightarrow 0} \frac{\log \mu(B(x, r))}{-\log r} \quad \text { and } \quad \bar{d}_{\mu}(x)=\varlimsup_{r \rightarrow 0} \frac{\log \mu(B(x, r))}{-\log r}
$$

where $B(x, r)$ denotes the open ball of radius $r$ centered in $x$. The measure $\mu$ is called exact dimensional if there is a constant $d$ such that

$$
\underline{d}_{\mu}(x)=\bar{d}_{\mu}(x)=d \quad \mu \text {-a.e. }
$$

One can show that $\underline{d}_{\mu}(x) \leq \operatorname{dim}_{H} \mu$ for $\mu$-almost every $x \in X$ and in particular if $\mu$ is exact dimensional then $\operatorname{dim}_{H} \mu=d$ (see $[\mathbf{Y}]$ ). 
We now give the definitions of some used quantitative indicators of recurrence.

First, let $s \geq 0$ be a real number and define

$$
\nu_{s}(x):=\varliminf_{n \rightarrow \infty} n^{s} \cdot d\left(x, T^{n} x\right)
$$

Given a positively recurrent point $x \in X$ let us define the sequence $\tau_{n}$ of closest returns as the subsequence of the integers which makes $d\left(x, T^{n} x\right)$ monotonically decreasing:

$$
\tau_{n}(x):=\inf \left\{k>\tau_{n-1}: d\left(x, T^{k} x\right) \leq d\left(x, T^{\tau_{n-1}} x\right)\right\}, \quad n>1,
$$

with $\tau_{1}$ chosen arbitrarily. Put moreover

$$
d_{n}(x):=d\left(x, T^{\tau_{n}} x\right)
$$

so that

$$
\nu_{s}(x)=\varliminf_{n \rightarrow \infty} \tau_{n}(x)^{s} \cdot d_{n}(x) .
$$

Boshernitzan proved that given $(X, \mathcal{B}, \mu, d, T)$ as above if the $\alpha$-dimensional Hausdorff measure $m_{\alpha}$ agrees with $\mu$ on the $\sigma$-algebra $\mathcal{B}$ then

$$
\nu_{\frac{1}{\alpha}}(x) \leq 1, \quad \mu \text {-a.e. }
$$

If instead $m_{\alpha}(X)=0$ then $\nu_{\frac{1}{\alpha}}(x)=0 \mu$-a.e. ([B] , Thm 1.2). An improvement has been obtained in $\mathrm{BS}$. Define the return time of a point $x \in X$ into the open ball $B(x, r)$ by

$$
\tau(x, r)=\inf \left\{k>0: T^{k} x \in B(x, r)\right\}=\inf \left\{k>0: d\left(x, T^{k} x\right)<r\right\}
$$

(note that the sequence $\tau_{n}$ defined above is but $\tau\left(x, d_{n-1}\right)$ ). The lower and upper pointwise recurrence rates of $x$ are defined as

$$
\underline{\mathcal{R}}(x, T)=\varliminf_{r \rightarrow 0} \frac{\log \tau(x, r)}{-\log r} \quad \text { and } \quad \overline{\mathcal{R}}(x, T)=\varlimsup_{r \rightarrow 0} \frac{\log \tau(x, r)}{-\log r} .
$$

Boshernitzan's result can then be rephrased by saying that $\underline{\mathcal{R}}(x, T) \leq \operatorname{dim}_{H} \mu$, $\mu$-a.e., whereas Barreira and Saussol proved that $\underline{\mathcal{R}}(x, T) \leq \underline{d}_{\mu}(x)$ and $\overline{\mathcal{R}}(x, T) \leq \bar{d}_{\mu}(x)$, $\mu$-a.e. (BS], Thm 1). In particular, if $T$ is a $\mathcal{C}^{1+\eta}$ diffeomorphism acting on a hyperbolic set and if $\mu$ is an ergodic equilibrium measure of a Hölder continuous weight function then $\underline{\mathcal{R}}(x, T)=\overline{\mathcal{R}}(x, T)=\operatorname{dim}_{H} \mu, \mu$-a.e. $([\mathrm{BS}]$, Thm 5$)$.

Let us consider a finite measurable partition $\mathcal{Z}$ of $X$. One may consider cylinder sets of the form

$$
A=Z_{n}(x) \in \vee_{k=0}^{n-1} T^{-k} \mathcal{Z}, \quad x \in Z_{n}(x) .
$$

The partition $\mathcal{Z}$ allows us to construct a coding map $\pi: X \rightarrow \Sigma_{\mathcal{Z}}$ onto a symbolic space $\Sigma_{\mathcal{Z}}$ which semiconjugates $T$ with the shift $S: \Sigma_{\mathcal{Z}} \rightarrow \Sigma_{\mathcal{Z}}$, i.e. $S \circ \pi=\pi \circ T$. Endowing $\Sigma_{\mathcal{Z}}$ with the pseudo metric

$$
d\left(\omega, \omega^{\prime}\right)=\sum_{k \geq 0} e^{-k}\left|\omega_{k}-\omega_{k}^{\prime}\right|
$$


we have that if $y, z \in Z_{n}(x)$ then $d(\pi(y), \pi(z)) \leq C e^{-n}$. Let us denote by $d_{\mathcal{Z}}$ the metric induced on $X$ by $d$ via the map $\pi$, and let $h_{\mu}(T, \mathcal{Z})$ be the $\mu$-entropy of $T$ with respect to the partition $\mathcal{Z}$. An easy consequence of the Shannon-McMillan-Breiman Theorem (see CFS, p. 257) is that the Hausdorff dimension of $\mu$ with respect to the metric $d_{\mathcal{Z}}$ coincides with $h_{\mu}(T, \mathcal{Z})$. Thus, in a sense, the results mentioned above can be viewed as generalizations of a theorem by Ornstein and Weiss ( $[\mathrm{OW}$, Thm 1) which in our language can be reformulated as follows: given a measurable transformation $T: X \rightarrow X$, a measurable partition $\mathcal{Z}$ of $X$ and a $T$-invariant ergodic probability measure $\mu$ on $X$, if we endow $X$ with the metric $d_{\mathcal{Z}}$ then $\underline{\mathcal{R}}(x, T)=\overline{\mathcal{R}}(x, T)=h_{\mu}(T, \mathcal{Z})$, $\mu$-a.e.

So far we considered recurrence indicators measuring how fast an orbit comes back near to the initial point or in a set containing the initial point. Another kind of indicator measures how fast the orbit of a given initial point approaches some other target point. This is interesting when we need to understand how close the orbit of $x$ approaches some singular locus of $(X, T)$ (for example the discontinuity set of $T$ ).

In the following theorem we fix a set of target points $x_{i} \in X, i=1, \ldots, l$ and study the entrance times into the balls $B\left(x_{i}, r\right)$. This result will be used in Section 4.4 to estimate the type of initial condition sensitivity of a class of discontinuous maps, the Interval Exchange Transformations.

Theorem 2.1. Let $(X, \mathcal{B}, \mu, d, T)$ be as above and $x_{1}, \ldots, x_{l}$ be a finite set of points in $X$. If $\alpha>\max \left\{\bar{d}_{\mu}\left(x_{1}\right)^{-1}, \ldots, \bar{d}_{\mu}\left(x_{l}\right)^{-1}\right\}$ then for $\mu$-almost all $x \in X$ it holds

$$
\varliminf_{n \rightarrow \infty} n^{\alpha} \min _{i} d\left(x_{i}, T^{n} x\right)=\infty .
$$

The proof follows straightforward from the following

Lemma 2.1. Let $(X, \mathcal{B}, \mu, d, T)$ be as above, $x_{i} \in X$ and assume that $\exists d$ and $\bar{r}>0$ such that $\mu\left(B\left(x_{i}, r\right)\right) \leq r^{d}$ for each $r<\bar{r}$. If $\alpha>\frac{1}{d}$ then for $\mu$-almost every $x \in X$ we have

$$
\varliminf_{n \rightarrow \infty} n^{\alpha} \cdot d\left(x_{i}, T^{n} x\right)=\infty
$$

Proof. Let us consider the family of sets $I_{n}\left(x_{i}\right)=T^{-n}\left(B\left(x_{i}, n^{-\alpha}\right)\right)$. From the fact that $\mu\left(B\left(x_{i}, r\right)\right) \leq r^{d}$ and $\alpha>\frac{1}{d}$ it follows that $\sum_{n} \mu\left(B\left(x_{i}, n^{-\alpha}\right)\right)<\infty$. Since $\mu$ is $T$-invariant then $\sum_{n} \mu\left(I_{n}\left(x_{i}\right)\right)<\infty$. Now each point $x$ such that

$$
\varliminf_{n \rightarrow \infty} n^{\alpha} \cdot d\left(x_{i}, T^{n} x\right)<1
$$

belongs to infinitely many sets in the family $I_{n}\left(x_{i}\right)$. By the Borel-Cantelli Lemma this implies that $x$ is contained in a set of zero measure.

So long for pointwise-like results. In a slightly different perspective we may consider a dynamical system $(X, T)$ where $T$ is a measurable transformation along with the set $M(X)$ of $T$-invariant Borel probability measures on $X$ and the set $\operatorname{EM}(X)$ of ergodic 
$T$-invariant Borel probability measures on $X$. Taking $\mu \in M(X)$ one can study the recurrence times into a given subset $A \subset X$ of positive $\mu$-measure. The basic result is Poincaré Recurrence Theorem which says that $\mu$-almost every point $x \in A$ returns to $A$ infinitely often, that is $\#\left\{n>0: T^{n} x \in A\right\}=\infty$.

Given $A$ as above we define its Poincaré recurrence time $\tau(A)$ as

$$
\tau(A)=\min \left\{n>0: T^{n} A \cap A \neq \emptyset\right\}=\inf \{\tau(x, A): x \in A\}
$$

where $\tau(x, A)=\inf \left\{k>0: T^{k} x \in A\right\}$ is the return time function in $A$. Poincaré Recurrence Theorem yields for $\mu \in M(X)$

$$
\mu(A)>0 \Longrightarrow \tau(A)<\infty .
$$

On the other hand we have the Kac's formula (see [Pe])

$$
\int_{A} \tau(x, A) d \mu(x)=1
$$

Therefore (2.13) can be improved to

$$
\mu(A)>0 \Longrightarrow \tau(A) \leq \frac{1}{\mu(A)} .
$$

Note that Poincaré recurrence for a set $A$ can be very different from typical return times $\tau(x, A)$. In the sequel we shall consider cylinder sets of the form (2.12). In this case, as we have seen, the return time $\tau\left(x, Z_{n}(x)\right)$ for a $\mu$-generic point $x$ behaves as $e^{n h_{\mu}(T, \mathcal{Z})}$ where $h_{\mu}(T, \mathcal{Z})$ is the entropy of $\mu$ w.r.t the partition $\mathcal{Z}$. On the other hand $\tau\left(Z_{n}(x)\right)$ is $\mathcal{O}(n)$.

An elementary argument presented in [STV] shows that the cylinder $Z_{n}(x)$ is completely determined by its first $\tau\left(Z_{n}(x)\right)$ symbols. More specifically, setting $Z_{n}(x)=$ $\left[\omega_{0} \omega_{1} \ldots \omega_{n-1}\right]$ and $\tau \equiv \tau\left(Z_{n}(x)\right)$ then if $j$ denotes the integer defined by $j \tau<n \leq$ $(j+1) \tau$ we have

$$
\omega_{i \tau}, \ldots, \omega_{(i+1) \tau-1}=\omega_{0}, \ldots, \omega_{\tau-1} \quad \text { for } \quad i=0,1, \ldots, j
$$

This suggests that the 'effective length' of the string $\omega_{0} \omega_{1} \ldots \omega_{n-1}$ is somehow encoded by the numerical sequence $\tau\left(Z_{n}(x)\right)$. We may also define the lower and upper $\mathcal{Z}$-recurrence rates as

$$
\bar{R}(x, T, \mathcal{Z})=\varlimsup_{n \rightarrow \infty} \frac{\tau\left(Z_{n}(x)\right)}{n} \text { and } \underline{R}(x, T, \mathcal{Z})=\varliminf_{n \rightarrow \infty} \frac{\tau\left(Z_{n}(x)\right)}{n} .
$$

We remark that this quantity is substantially different from that defined in eq. (2.11), that is a ratio of logarithms to extract the power law behaviour of the recurrence times with respect to the distance. Moreover the quantity defined by Equation (2.11) does not depend on the choice of a partition.

We now collect some results about this recurrence rate which will be useful in what follows. 


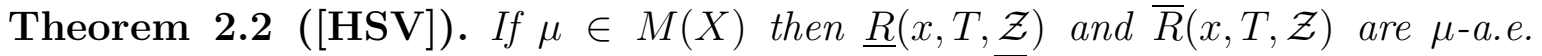
invariant. If moreover $\mu \in E M(X)$ then $\underline{R}(x, T, \mathcal{Z})$ and $\bar{R}(x, T, \mathcal{Z})$ are $\mu$-a.e. constant.

Theorem 2.3 ([STV]). If $\mu \in E M(X)$ and $h_{\mu}(T, \mathcal{Z})>0$ then $\underline{R}(x, T, \mathcal{Z}) \geq 1$, $\mu$-a.e.

Remark 1. We recall that the pair $(X, T)$ is said to have the specification property if the following holds: for any $\epsilon>0$, there exists a positive integer $M(\epsilon)$ s.t. for any $k \geq 2$ and for any $k$ points $x_{1}, \ldots, x_{k} \in X$, and for any positive integers $a_{1} \leq$ $b_{1}<a_{2} \leq b_{2}<\cdots a_{k} \leq b_{k}$ satisfying $a_{i}-b_{i-1} \geq M(\epsilon)$ for $2 \leq i \leq k$, and for any $p \geq M(\epsilon)+b_{k}-a_{1}$, there exists a point $x \in X$ with $T^{p} x=x$ such that $d\left(T^{n} x, T^{n} x_{i}\right) \leq \epsilon$ for any $a_{i} \leq n \leq b_{i}, 1 \leq i \leq k .(X, T)$ is said to be weakly specified if the above holds for $k=2$. One easily sees that if $(X, T)$ is weakly specified then $\tau\left(Z_{n}(x)\right) \leq n$ and therefore $\underline{R}(x, T, \mathcal{Z})=\bar{R}(x, T, \mathcal{Z})=1$, $\mu$-a.e.

Remark 2. Theorem 2.3 turns out to be a key estimate in proving exponential and Poisson statistics for return times in dynamical systems [HSW]. In the following, however, we shall take a different direction, by comparing the typical behaviour of $\tau\left(Z_{n}(x)\right)$ with the algorithmic information of the orbit of $x$ obtaining results which are meaningful in the null entropy case. These results have the same philosophy of Theorem 2.3: if the information rate is big then the recurrence must be big as well.

\subsection{Algorithmic information content}

Let us briefly recall the notion of Algorithmic Information Content (AIC) of orbits in dynamical systems with respect to some given partition.

To this end one starts with a Turing machine $A$ and a finite string $s$ written in some finite alphabet $\mathcal{A}$. Let $\Sigma$ be the set of finite binary strings. Let $p \in \Sigma, p$ will be considered as a program to be run. If we start the machine $A$ with input $p$, the computation stops and the output is $s$, we write $A(p)=s$. By this notation we emphasize the function: input $\rightarrow$ output that is naturally associated to the machine. A function is said to be recursive if its values can be computed by a Turing machine as above. Some computing machine stops and give an output for each given input string. Some other has a set of inputs that leads to a computation that never stops. In this case the output is not defined.

In the first case we say that the machine defines a total recursive function (from $\Sigma$ to the set of finite strings in $\mathcal{A}$ ). In the second case to the machine it can be associated a function that is defined only on a subset of $\Sigma$ in this case we say that $A$ defines a partial recursive function (from $\Sigma$ to another set of strings). Let us denote by $\ell(p)$ the length of $p$.

The Kolmogorov complexity or Algorithmic Information Content of $s$ relative to A is the quantity

$$
A I C_{A}(s)=\min _{p: A(p)=s} \ell(p)
$$

If there is no $p$ s.t. $A(p)=s$ then we put $A I C_{A}(s)=\infty$.

There are countably many Turing machines which may be computably enumerated as $A_{1}, A_{2}, \ldots$ For $n \in \mathbb{N}$ let $e_{n}$ be the $n$-th binary string in the lexicographical order 
$0,1,00,01,10,11,000, \ldots$ so that $\ell\left(e_{n}\right) \leq \log _{2} n$. We say that a Turing machine $U$ is universal if for any $n \in \mathbb{N}$ and any finite $0-1$ string $p$ we have $U\left(\hat{e}_{n} p\right)=A_{n}(p)$ where, for a given word $q$ of length $m$, we have denoted by $\hat{q}$ the word $q(0) q(0) q(1) q(1) \ldots q(m-$ 1) $q(m-1) 01$. This in particular means that if $A$ is any Turing machine a constant $C_{A}$ can be found so that for any finite binary string $s$ we have $A I C_{U}(s) \leq A I C_{A}(s)+C_{A}$. Then the AIC is independent of the choice of the universal machine $A$ up to a constant. Since we are considering the asymptotical behaviour of the AIC for very long strings this constant is not relevant.

We can now define an (upper) average complexity of an infinite sequence $\omega=$ $\omega_{0} \omega_{1} \ldots$ as the limit

$$
\bar{K}_{U}(\omega)=\varlimsup_{n \rightarrow \infty} \frac{A I C_{U}\left(\omega_{0} \ldots \omega_{n-1}\right)}{n}
$$

and a corresponding lower complexity $\underline{K}_{U}(\omega)$ with $\varlimsup$ im replaced by $\underline{\lim }$.

Coming back to our dynamical system $(X, T)$ and given a point $x \in X$ we may define the Algorithmic Information Content $A I C(y, n)$ of any piece of orbit $\left\{y, T y, \ldots, T^{n-1} y\right\}$ with $y \in Z_{n}(x)=\left[\omega_{0} \omega_{1} \ldots \omega_{n-1}\right]$ just as $A I C(y, n) \equiv A I C_{U}\left(\omega_{0} \ldots \omega_{n-1}\right)$. In particular one defines the upper and lower algorithmic complexities of a point $x$ w.r.t. $T$ and $\mathcal{Z}$ as $\bar{K}(x, T, \mathcal{Z})=\bar{K}_{U}(\omega)$ and $\underline{K}(x, T, \mathcal{Z})=\underline{K}_{U}(\omega)$, respectively, where $\omega$ is the (infinite) symbolic coding of the orbit of $x$ with $T$ on the partition $\mathcal{Z}$. We have the following result

Theorem $2.4([\mathbf{B r}],[\mathbf{W}])$. If $\mu \in E M(X)$ then $\underline{K}(x, T, \mathcal{Z})=\bar{K}(x, T, \mathcal{Z})=h_{\mu}(T, \mathcal{Z})$, p-a.e.

The above theorem shows a strict relation between AIC and entropy. Such a relation is also intuitive since both entropy and $K_{U}$ measures the information rate of the symbolic strings that comes from the given partition. The difference between this two notions is that entropy is defined using the Shannon information while $K_{U}$ uses the algorithmic information. Moreover, entropy is a global (average) notion while $K_{U}$ is pointwise. The entropy and thus the increase of the information has a crucial role in the study of chaotic dynamics and it is related with recurrence, dimension, initial condition sensitivity. By the above theorem also the algorithmic information of symbolic orbits is related to all these indicators of chaos.

In the case where entropy is null the information that is necessary to describe the orbit increases less than linearly. There are many possible such asymptotic behaviours. In this case then the asymptotic behaviour of the algorithmic information that is necessary to describe the orbit gives an orbit complexity indicator more refined than $K_{U}$ (that is almost always null). This information behaviour is also related to dimension and initial condition sensitivity. Recent results in Ga proves quantitative relations between dimension, AIC and initial conditions sensitivity that are meaningful in the zero entropy case.

In the following we start to investigate also some relation with recurrence. We will see that certain recurrence rates implies strong limitations to the algorithmic information of the orbits. In Section 4.4 we will also see how by the recurrence near given 
points it is possible to have an estimation of the initial condition sensitivity of a system, and then of the algorithmic information of its orbits.

As noted above (Eq.(2.16) ) the AIC of any $n$-long orbit of points in $Z_{n}(x)=$ $\left[\omega_{0} \omega_{1} \ldots \omega_{n-1}\right]$ is bounded by the AIC of the initial word $\omega_{0} \ldots \omega_{\tau-1}$ plus the AIC needed to repeat this word up to the size $n$ of the cylinder, that is

$$
A I C(x, n) \leq A I C\left(x, \tau\left(Z_{n}(x)\right)\right)+\log n+C
$$

To construct the symbolic string that gives the $n$ steps symbolic orbit of $x$ with respect to $\mathcal{Z}$ we need the information that is necessary for the first period (that is $\tau\left(Z_{n}(x)\right)$ steps long) and the information needed for $n$ (that is about $\log n$ bits).

If, moreover, $(X, T)$ is weakly specified then $\tau\left(Z_{n}(x)\right) \leq n$ so that we also have

$$
A I C\left(x, \tau\left(Z_{n}(x)\right)\right) \geq A I C(x, n) .
$$

Therefore, in this case the condition $h_{\mu}(T, \mathcal{Z})>0$ entails $^{1}$

$$
A I C(x, n) \sim h_{\mu}(T, \mathcal{Z}) \tau\left(Z_{n}(x)\right) \sim h_{\mu}(T, \mathcal{Z}) n, \quad \mu-\text { a.e. }
$$

On the other hand, by Theorem 2.4 the condition $h_{\mu}(T, \mathcal{Z})=0$ implies $\underline{K}(x, T, \mathcal{Z})=$ $\bar{K}(x, T, \mathcal{Z})=0, \mu$-a.e. and therefore

$$
A I C(x, n)=o(n), \quad \mu-a . e .
$$

Can we say something about the asymptotic relations between $A I C(x, n)$ and $\tau\left(Z_{n}(x)\right)$ in this case? We start proving the following results that are in some sense extensions of the Theorem 2.3 for the null entropy case. We include the case of systems with an infinite invariant measure, assuming conservativity of the systems, a classical assumption in these cases that is verified for systems with a probability invariant measure. The extension to infinite measures will prove useful in Section 3.

Theorem 2.5. Let $(X, T, \mu)$ be an ergodic conservative dynamical system, with $\mu$ a $T$-invariant and not necessarily finite measure on $X$. Then

$$
\bar{\alpha}(x):=\inf \left\{\alpha: \varliminf_{n \rightarrow \infty} \frac{\tau\left(Z_{n}(x)\right)}{n^{\alpha}}=0\right\}
$$

is $\mu$-a.e. equal to a constant $\bar{\alpha}$. If moreover $0 \leq \bar{K}(x, T, \mathcal{Z})<\infty$ then for $\mu$-a.e. $x \in X$ we have

$$
\varliminf_{n \rightarrow \infty} \frac{A I C(x, n)}{n^{\beta}}=0, \quad \forall \beta>\bar{\alpha} .
$$

\footnotetext{
${ }^{1}$ Notations: Here and in the sequel, for two sequences $a_{n}$ and $b_{n}$ we shall write $a_{n} \sim b_{n}$ if the quotient $a_{n} / b_{n}$ tends to unity as $n \rightarrow \infty$. Moreover, the notation $a_{n} \approx b_{n}$ means that $a_{n} / b_{n}=o\left(n^{\epsilon}\right)$ as well as $b_{n} / a_{n}=o\left(n^{\epsilon}\right)$ for $n \rightarrow \infty$ and $\forall \epsilon>0$. This condition is satisfied if $a_{n} / b_{n} \sim L(n)$ where $L(n)$ is some function slowly varying at infinity, i.e. $L(c n) \sim L(n)$ for every positive $c$. Both $\sim$ and $\approx$ are equivalence relations and we shall denote by $\left[a_{n}\right]$ and $\left[\left[a_{n}\right]\right]$ respectively, the equivalence classes of $a_{n}$ w.r.t to these relations.
} 
Proof. The constancy of $\bar{\alpha}(x) \mu$-a.e. follows at once from Theorem 2.2 . Using (2.18) we have, for $\beta>\bar{\alpha}$,

$$
\begin{aligned}
0 & \leq \varliminf_{n \rightarrow \infty} \frac{A I C(x, n)}{n^{\beta}} \leq \varliminf_{n \rightarrow \infty}\left(\frac{A I C\left(x, \tau\left(Z_{n}(x)\right)\right)}{n^{\beta}}+\frac{\log n}{n^{\beta}}\right) \\
& \leq \varlimsup_{n \rightarrow \infty} \frac{A I C\left(x, \tau\left(Z_{n}(x)\right)\right)}{\tau\left(Z_{n}(x)\right)} \cdot \varliminf_{n \rightarrow \infty} \frac{\tau\left(Z_{n}(x)\right)}{n^{\beta}}=\bar{K}(x, T, \mathcal{Z}) \cdot 0=0
\end{aligned}
$$

where we recall that if $\mu(X)<\infty$ then by Theorem 2.4 it holds $h_{\mu}(T, \mathcal{Z})=\bar{K}(x, T, \mathcal{Z})$, $\mu$-a.e. If instead $\mu(X)=+\infty$ then $\mu$-a.e. it holds $\bar{K}(x, T, \mathcal{Z})=0$ (see [B] Thm. $3.3)$.

From this theorem we may deduce some consequences.

Corollary 2.1. Let $\mu \in E M(X)$ and suppose that for $\mu$-a.e. $x \in X$ we have $\bar{\alpha}(x)=$ $\bar{\alpha}<1$. Then $h_{\mu}(T, \mathcal{Z})=0$. If moreover $\bar{\alpha}=0$ then $A I C(x, n) \approx$ constant $\mu$-a.e.

We remark that the first part of this statement is contained in Theorem 2.3.

If $\bar{R}(x, T, \mathcal{Z})<1$ the system is forced to have a strong constraint about the information content of its orbits, as it is shown in the following theorem:

Theorem 2.6. If $\bar{R}(x, T, \mathcal{Z})<1$ then $A I C(x, n) \approx$ constant. More specifically we have

$$
\varlimsup_{n \rightarrow \infty} \frac{\operatorname{AIC}(x, n)}{\log ^{2} n} \leq \frac{-1}{\log \bar{R}(x)} .
$$

The proof of this theorem consists in a straightforward application to Eq2.18 of the following lemma with the identifications $d=1 / \bar{R}$ and $a_{n}=A I C(x, n)$.

Lemma 2.2. Let $a_{n}$ be a real sequence such that $\exists d>1$ and $\bar{n}$ such that $\forall n>\bar{n}$ there is $d_{n}$ such that $d_{n} \geq d, \frac{n}{d_{n}} \in \mathbb{N}$ and $a_{n} \leq a_{\frac{n}{d_{n}}}+\log n+C$. Then

$$
\varlimsup_{n \rightarrow \infty} \frac{a_{n}}{\log ^{2} n} \leq \frac{1}{\log d}
$$

Proof. Set $A=\max _{n \leq \bar{n}} a_{n}$ and $f: \mathbb{N} \rightarrow \mathbb{N}$ be the map given by $f(n)=\frac{n}{d_{n}}$. Note that $f(n)<n$. Letting $n>\bar{n}$ we have

$$
a_{n} \leq a_{f(n)}+\log n+C \leq a_{f^{2}(n)}+\log n+\log f(n)+2 C \leq \cdots \leq a_{f^{k}(n)}+k(C+\log n)
$$

with $k=\min \left\{i \in \mathbb{N}: f^{i}(n) \leq \bar{n}\right\}$. One readily realizes that $k \leq \frac{\log (n / \bar{n})}{\log d}$. Therefore $a_{n} \leq A+\frac{\log (n / \bar{n})}{\log d}(C+\log n)$.

We remark that the above result is pointwise and does not depend on the invariant measure. We will see an application of Theorems 2.5 and 2.6 in the next section for dynamical systems with an infinite invariant measure absolutely continuous with respect to the Lebesgue measure.

Of course Theorem [2.5] is interesting when $\bar{\alpha}<1$. When $\bar{\alpha}=1$ the conclusion of Theorem 2.5 is not so interesting. However, as we shall see below (Theorem 
4.9) there are uniquely ergodic dynamical systems with zero entropy and such that $0<\underline{R}(x, T, \mathcal{Z}) \leq \bar{R}(x, T, \mathcal{Z}) \leq 1$ (and thus $\bar{\alpha} \geq 1$ ). By the way, this provides a counterexample to show that the inequality in the above theorem cannot be reversed in general. There are systems with very low orbit complexity and several possible recurrence rates. We are now going to discuss some examples.

\section{Markov interval maps}

In this Section we apply the results of the previous one to calculate recurrence times for a class of intermittent maps on the interval, with finite or infinite absolutely continuous invariant measure.

Roughly speaking, a map $T:[0,1] \rightarrow[0,1]$ is called Markov if there is a finite partition $\mathcal{Z}$ of $[0,1]$ such that the image of each of its elements is the union of some of its elements.

Let us consider a map $T$ such that

1) $T$ is increasing and $\mathcal{C}^{2}$ restricted to each interval of the partition $\mathcal{Z}$;

2) $T$ is onto on each interval of the partition.

All maps satisfying 1) and 2) are obviously Markov. We remark that all the maps that have properties 1) and 2) are topologically conjugate, but the conjugation may be not absolutely continuous and then some properties which are related to the Lebesgue measure may change.

Let us consider the partition $\mathcal{Z}=\{[0,1 / 2),[1 / 2,1]\}$. The map $\pi:[0,1] \rightarrow\{0,1\}^{\mathbb{N}}$ defined by $\pi(x)=\omega_{0} \omega_{1} \omega_{2} \ldots$ according to $T^{j} x \in I_{\omega_{j}}$ for $j \geq 0$ is a coding map which is a homeomorphism on the residual set of points in $[0,1]$ which are not preimages of 1 . Moreover $S \circ \pi=\pi \circ T$ where $S$ denotes the right shift map on $\{0,1\}^{\mathbb{N}}$. It is plain that there are exactly $2^{n}$ cylinders of the form $Z_{n}(x)=\left[\omega_{0}, \omega_{1}, \ldots, \omega_{n-1}\right]$ and each of them contains exactly one periodic point of prime period $n$. This implies at once $\tau\left(Z_{n}(x)\right) \leq$ $n$ for every $x$. Now, if $T$ is uniformly expanding, i.e. $\exists \rho>1$ such that $T^{\prime}(x) \geq \rho$ for all $x \in[0,1]$ then it is well known (see $[\mathrm{KH}]$ ) that $T$ possesses a unique (ergodic) absolutely continuous invariant measure $\mu$ with $h_{\mu}(T)=h_{\mu}(T, \mathcal{Z})>0$. Putting together the above, Theorem [2.3 and Remark [1, we get $\underline{R}(x, T, \mathcal{Z})=\bar{R}(x, T, \mathcal{Z})=1, \mu-$ a.e. This condition continues to hold for the intermittent situation where $T^{\prime}(0)=1$ provided the invariant measure $\mu$ is finite (so that the entropy is still positive).

In the opposite case (i.e. for example when $T^{\prime}(0)=1$ and $T^{\prime \prime}(0)=0$ ) we have no absolutely continuous invariant probability measure. In such case in principle we only have the inequalities $\underline{R}(x, T, \mathcal{Z}) \leq \bar{R}(x, T, \mathcal{Z}) \leq 1, \mu-$ a.e.. In this case however, if the map satisfies some weak auxiliary assumptions, $\bar{R}$ can be estimated.

Proposition 3.1. Under the assumptions 1) and 2), if $T$ is such that $T^{\prime}(0)=1$ and $T^{\prime \prime}(x) \sim x^{z-2}$ as $x \rightarrow 0$, for some $z>2$, and there exists an absolutely continuous infinite invariant measure, then $\bar{R}(x, T, \mathcal{Z})=1$ Lebesgue - a.e.

Proof. First, we can use the results of $[\mathrm{Pr}]$ to find out a $C^{1}$-diffeomorphism of $[0,1]$ which conjugates $T$ with the map $T_{z}(x)=x+x^{z}(\bmod 1)$. In turn, the latter (called Manneville map) is absolutely continuously conjugated to its piecewise linear versions 
that can be studied by the theory of recurrent events (see [BoGa]), obtaining that $A I C(x, n)$, with respect to the partition $\mathcal{Z}$, increases for $z>2$ as a power law $n^{\alpha}$, with $\alpha=\frac{1}{z-1} \in(0,1)$, and that for Lebesgue a.e. $x$ it holds

$$
\varliminf_{n \rightarrow \infty} \frac{A I C(x, n)}{n^{\beta}}=+\infty \quad \forall \beta<\alpha
$$

Hence if $\bar{R}(x, T, \mathcal{Z})<1$ on a set of positive Lebesgue measure, then by Theorem 2.6. $A I C(x, n) \approx$ constant on this set, and this would contradict Equation (3.22).

An analogous result can be obtained for the index $\bar{\alpha}$ of Theorem 2.5 .

Proposition 3.2. Under the same hypothesis of Proposition 3.1 it holds $\bar{\alpha} \geq \frac{1}{z-1}$.

Proof. By contradiction, if $\alpha<\frac{1}{z-1}$, then by Theorem 2.5] it follows that Equation (3.22) cannot be verified.

\section{Ergodic piecewise isometries}

\subsection{Recurrence results for ergodic rotations}

Let $X=(0,1]$ be the unit circle and $\mu$ the Lebesgue measure. Let moreover $\alpha \in(0,1]$ and $T: X \rightarrow X$ be the rigid translation

$$
T(x)=x+\alpha(\bmod 1) .
$$

The measure $\mu$ is clearly $T$-invariant. If $\alpha \in \mathbb{R} \backslash \mathbb{Q}$ all orbits are dense and $\mu$ is the unique (and thus ergodic) invariant measure, which is obviously exact dimensional with $d=1$ (cfr. (2.4) $)$. Let us consider the partition $\mathcal{Z}=\left(A_{0}, A_{1}\right)$ of $X$ into the half-open $\operatorname{arcs} A_{0}=[0,1 / 2)$ and $A_{1}=[1 / 2,1)$. For $\alpha$ irrational $T$ is a minimal homeomorphism of $X$. Therefore $\mathcal{Z}$ is generating for $T$ and the partition $\mathcal{Z}^{n}=\mathcal{Z} \vee T^{-1} \mathcal{Z} \vee \cdots \vee T^{-n+1} \mathcal{Z}$ is made out of $2 n$ arcs. This can be easily realized by induction: when passing from $\mathcal{Z}^{n-1}$ to $\mathcal{Z}^{n}$ one has to add to the endpoints of the arcs belonging to $\mathcal{Z}^{n-1}$ the two new points $T^{-n}(0)$ and $T^{-n}(1 / 2)$. Thus,

$$
0 \leq h_{\mu}(T, \mathcal{Z}) \leq \lim _{n \rightarrow \infty} \frac{\log (2 n)}{n}=0 .
$$

It is interesting to note that the lengths of the arcs of $\mathcal{Z}^{n}$ can actually take only five values (see $\mathrm{AB}$, Thm 18).

Note moreover that $(X, T)$ is metrically isomorphic to the subshift given by the closure of $\pi([0,1))$ where the coding map $\pi:[0,1] \rightarrow\{0,1\}^{\mathbb{N}}$ is given by $\pi(x)_{n}=$ $\chi_{A_{1}}\left(T^{n}(x)\right)$. Let

$$
\alpha=\frac{1}{a_{1}+\frac{1}{a_{2}+\frac{1}{a_{3}+\cdots}}} \equiv\left[a_{1}, a_{2}, a_{3}, \ldots\right]
$$


be the continued fraction expansion of $\alpha$ and $p_{n} / q_{n}=\left[a_{1}, a_{2}, \ldots, a_{n}\right]$ the sequence of its rational approximants. It is well known that (see [RS])

$$
\frac{1}{q_{n} \cdot\left(q_{n+1}+q_{n}\right)}<\left|\alpha-\frac{p_{n}}{q_{n}}\right|<\frac{1}{q_{n} \cdot q_{n+1}} .
$$

From the estimate (4.25) one deduces the following characterization for the denominators $q_{n}$ : let $\|x\|=\min \{|x-p|: p \in \mathbb{Z}\}$ be the distance from the nearest integer, then

$$
q_{n}=\min \left\{k>q_{n-1}:\|k \alpha\|<\left\|q_{n-1} \alpha\right\|\right\}
$$

so that $\|k \alpha\| \geq\left\|q_{n-1} \alpha\right\|$ for all $k<q_{n}$. Also notice that

$$
d\left(x, T^{k} x\right)=\min _{p \in \mathcal{X}}|x+k \alpha-x-p|=\|k \alpha\|
$$

for all $x \in X$. Now set

$$
f_{n}:=d\left(x, T^{q_{n}} x\right)=\left\|q_{n} \alpha\right\|=\left|q_{n} \alpha-p_{n}\right|=(-1)^{n}\left(q_{n} \alpha-p_{n}\right)
$$

It holds

$$
f_{n-1}=a_{n+1} f_{n}+f_{n+1}
$$

and also

$$
f_{n}=\prod_{k=0}^{n} G^{k}(\alpha)
$$

where $G:[0,1] \rightarrow[0,1]$ is the Gauss transformation given by $G(x)=\{1 / x\}$ for $x>0$ and $G(0)=0$. Equation (4.30) shows that for all irrational $0<\alpha<1$ the sequence $f_{n}$ is strictly decreasing and by (4.25) it satisfies the bounds

$$
\frac{1}{a_{n+1}+2}<q_{n} \cdot f_{n}<\frac{1}{a_{n+1}}
$$

Definition 4.1. We shall say that the irrational number $\alpha$ is of type $\gamma$ if

$$
\gamma=\sup \left\{\beta>0 / \varliminf_{k \rightarrow \infty} k^{\beta} \cdot\|k \alpha\|=0\right\} .
$$

By (4.26) one has that if $q_{n} \leq k<q_{n+1}$ then $k^{\beta} \cdot\|k \alpha\| \geq q_{n}^{\beta} \cdot\left\|q_{n} \alpha\right\|$ and the lower limit in (4.32) is reached along the subsequence $k=q_{n}$. In addition by (4.31) we have $q_{n}^{1-\epsilon} \cdot\left\|q_{n} \alpha\right\|<1 / q_{n}^{\epsilon}$ and therefore $\gamma \geq 1$. The set of type 1 numbers includes those with bounded partial quotients (see below, Proposition 4.9). One has (see [RS], cap. 4.54.6) $\underline{\lim }_{k \rightarrow \infty} k \cdot\|k \alpha\| \leq 1 / \sqrt{m^{2}+1}$ for any $\alpha=\left[a_{1}, a_{2}, \ldots\right]$ unless $a_{i}<m$ for all $i$ large enough. For the golden mean $\alpha=[1,1,1, \ldots]=\frac{\sqrt{5}-1}{2}$ we find $\underline{\lim }_{k \rightarrow \infty} k \cdot\|k \alpha\|=1 / \sqrt{5}$. On the contrary, we say that $\alpha$ is well approximated by rational numbers if $\eta(\alpha)>0$ where $\eta(\alpha)$ is the supremum of all $\eta$ such that for all $k \geq 1$ we have $\left\|q_{k} \alpha\right\| \leq C q_{k}^{-(1+\eta)}$. In this case $q_{k} \cdot\left\|q_{k} \alpha\right\| \leq C q_{k}^{-\eta(\alpha)}$ and $\alpha$ is of type $1+\eta(\alpha)$. If for example $a_{k}=2^{2^{k}}$ then $\alpha$ is of type 2 . 
Let us first dwell upon pointwise recurrence properties. First, Boshernitzan theorem mentioned in Section 2.1 along with minimality yield that the function $\nu_{1}(x)$ defined in (2.8) is finite for all $x$ and all $\alpha \in \mathbb{R} \backslash Q$, which is not very informative. We are now going to show that, in some sense, for ergodic rotations the role of the Hausdorff dimension is played by the type of the irrational number $\alpha$. First, from the general properties of continued fractions recalled above one readily gets the following

Theorem 4.7. Let $X=\mathbb{R} / \mathbb{X}$ and $T: X \rightarrow X$ be the rotation of an irrational angle $0<\alpha<1$. Then the quantities $\tau_{n}, d_{n}$ and $\nu_{s}$, defined in (2.6), (2.7) and (2.8), respectively, are constant on $X$ with $\tau_{n}=q_{n}$ and $d_{n}=f_{n}$. Moreover, if the irrational number $\alpha$ is of type $\gamma \geq 1$ then $\nu_{\gamma}<\infty$ and $\nu_{s}=0$ for $s<\gamma$.

In terms of the lower pointwise recurrence rate $\underline{\mathcal{R}}(x, T)$ defined in (2.11), the above result is equivalent to the following result

Theorem 4.8. If $\alpha$ is of type $\gamma \geq 1$ then $\underline{\mathcal{R}}(x, T)=1 / \gamma$.

Proof. Note that in this case the return time defined in (2.10) can be written as $\tau(x, r)=\inf \{k>0:\|k \alpha\|<r\}$. Recalling that the numbers $f_{n}$ are strictly decreasing, for each $r$ let $n$ be such that $f_{n}<r \leq f_{n-1}$. By (4.26) we have that for $0<k<q_{n}$ it holds $\|k \alpha\| \geq\left\|q_{n-1} \alpha\right\|=f_{n-1} \geq r$ but $\left\|q_{n} \alpha\right\|=f_{n}<r$. Therefore, for these values of $r$ we have $\tau(x, r)=q_{n}$. If moreover $\alpha$ is of type $\gamma$ then for $\epsilon>0$ and $k>0$ there is $C_{\epsilon}>0$ s.t. $k^{\gamma+\epsilon} \cdot\|k \alpha\| \geq C_{\epsilon}$. In particular choosing $k=q_{n}$ this gives

$$
q_{n}^{\gamma+\epsilon}>\frac{C_{\epsilon}}{f_{n}} \geq \frac{C_{\epsilon}}{r}
$$

Therefore $(\gamma+\epsilon) \log q_{n} \geq-\log r+\log C_{\epsilon}$ and

$$
\underline{\mathcal{R}}(x, T)=\underline{\lim } \frac{\log (\tau(x, r))}{-\log r}=\underline{\lim } \frac{\log q_{n}}{r \rightarrow 0} \geq \frac{1}{\gamma+\epsilon},
$$

whence $\underline{\mathcal{R}}(x, T) \geq 1 / \gamma$ by the arbitrariness of $\epsilon$.

In the other direction note that $\underline{\lim } k^{\gamma-\epsilon} \cdot\|k \alpha\|=0$. Therefore there is a subsequence $k_{j}$ tending to infinity so that $k_{j}^{\gamma-\epsilon} \cdot\left\|k_{j} \alpha\right\|<1$. Choose a sequence $r_{j}$ of $r$-values such that $r_{j}^{-1}<k_{j}^{\gamma-\epsilon} \leq 2 r_{j}^{-1}$. It then follows that $\left\|k_{j} \alpha\right\|<r_{j}$ and therefore $\tau\left(x, r_{j}\right) \leq k_{j} \leq$ $\left(2 / r_{j}\right)^{\frac{1}{\gamma-\epsilon}}$. Finally we get

$$
\frac{\log \tau\left(x, r_{j}\right)}{-\log r_{j}} \leq\left(\frac{\log 2-\log r_{j}}{-\log r_{j}}\right) \frac{1}{\gamma-\epsilon}
$$

Taking the limit $j \rightarrow \infty$ we get $\underline{\mathcal{R}}(x, T) \leq 1 / \gamma$ by the arbitrariness of $\epsilon$.

As far as the recurrence rates (2.17) are concerned we first mention a result obtained by Afraimovich, Chazottes and Saussol which in our language says that if $\alpha$ is of type $\gamma>3$ then $\underline{R}(x, T, \mathcal{Z})=0$ (see $[\mathrm{ACS}$, Thm 4.4).

Now, according to (4.27) the distance $d\left(x, T^{n} x\right)$ does not depend on $x$ but only on $n$ and $\alpha$, so that for a given (half-open) arc $A \subset X$ of length $|A|$ we have

$$
\tau(A)=\min \{r>0:\|r \alpha\|<|A|\} .
$$


Therefore for two half-open $\operatorname{arcs} A$ and $B$ we have

$$
|A|<|B| \Longrightarrow \tau(A) \geq \tau(B)
$$

A more precise information can be obtained from the three gap theorem (see [AB], Sec. 4). First, it says that if $A$ is such that $|A| \leq 1 / 2$ then $|A|$ can be expressed uniquely as

$$
|A|=c f_{k}+f_{k+1}+g \quad \text { for } \text { some } \quad k \geq 1, \quad 0<g \leq f_{k}, \quad 1 \leq c \leq a_{k+1} .
$$

Using (4.29) we see that the integer $k$ is determined by the inequalities

$$
f_{k}+f_{k+1}<|A| \leq f_{k-1}+f_{k}
$$

Second, the return time to the set $A$ can assume, on the points of $A$, at most three values, one being the sum of the other two: they are $r_{1}=q_{k}$ (with frequency $(c-$ 1) $\left.f_{k}+f_{k+1}+g\right), r_{2}=q_{k+1}-c q_{k}$ (with frequency $g$ ) and $r_{3}=r_{1}+r_{2}$ (with frequency $\left.f_{k}-g\right)$.

This yields the following

Lemma 4.3. Let $|A|$ be as in 4.35). Then

$$
\tau(A)= \begin{cases}q_{k}, & \text { if } c<a_{k+1}, \\ q_{k-1}, & \text { if } c=a_{k+1}\end{cases}
$$

ExAmple. If $|A|=f_{k-1}$ for some $k>1$ then using (4.29) we find $g=f_{k}, c=$ $a_{k+1}-1$ and $\tau(A)=q_{k}$. Note that the same results from (4.33) and (4.26). In this case the largest return time $r_{3}$ has frequency zero and therefore, by uniform distribution, it does not appear at all.

Theorem 4.9. Suppose that $\alpha=\left[a_{1}, a_{2}, \ldots\right]$ with $a_{i}=O(1), \forall i$, then $\underline{R}(x, T, \mathcal{Z})>0$ for all $x$.

Proof. By the five distance theorem (see [AB], Sec. 7) we know that the lengths of the intervals forming the partition $\mathcal{Z}^{n}$ take at most five values. In order to estimate the lengths of such intervals let us reason as follows. Let $\mathcal{P}^{n}$ and $\mathcal{Q}^{n}$ be the partitions of the circle by the points $\{j \alpha\}_{0 \leq j<n}$ and $\left\{\frac{1}{2}+j \alpha\right\}_{0 \leq j<n}$, respectively. Then we have $\mathcal{Z}^{n}=\mathcal{P}^{n} \vee \mathcal{Q}^{n}$. From the three distance theorem (see [BCF], Sec. 3) we know that for $q_{k}<n \leq q_{k+1}$ the intervals of the partitions $\mathcal{P}^{n}$ and $\mathcal{Q}^{n}$ have three possible lengths, which are given by: $f_{k}, c_{n} f_{k}+f_{k+1}$, for some $1 \leq c_{n} \leq a_{k+2}$, and $\left(1+c_{n}\right) f_{k}+f_{k+1}$ (this last one being not taken for some values of $n$, in particular for $n=q_{k+1}$ ). Therefore, for $q_{k}<n \leq q_{k+1}$ the intervals of $\mathcal{Z}^{n}$ have lengths not larger than $\left(1+c_{n}\right) f_{k}+f_{k+1}$.

Now, if $a_{i} \leq m$ then for $q_{k}<n \leq q_{k+1}$ the intervals of $\mathcal{Z}^{n}$ have lengths not larger than $(m+1) f_{k}+f_{k+1}$. Let $B$ be such that $|B|=m f_{k}+f_{k+1}+g$ with $0<g \leq f_{k}$. By the above, we can find $k^{\prime} \leq k$ with $k-k^{\prime}$ bounded uniformly in $k$, together with $0<g^{\prime} \leq f_{k^{\prime}}$ and $1 \leq c \leq a_{k^{\prime}+1}$ so that $|B|=c f_{k^{\prime}}+f_{k^{\prime}+1}+g^{\prime}$. Whence $\tau(B) \geq q_{k^{\prime}-1}$ by Lemma 4.3. Moreover, using (4.34) we see that $\tau(A) \geq q_{k^{\prime}-1}$ for any $A \in \mathcal{Z}^{n}$ with $q_{k}<n \leq q_{k+1}$. This yields

$$
\underline{R}(x, T, \mathcal{Z}) \geq \underline{\lim }_{k \rightarrow \infty} \frac{q_{k^{\prime}-1}}{q_{k+1}}>\varliminf_{k \rightarrow \infty} \prod_{j=k^{\prime}}^{k+1}\left(a_{j}+1\right)^{-1} \geq(m+1)^{k^{\prime}-k-1}>0
$$

where we have repeatedly used the inequality $q_{k+1}<\left(a_{k+1}+1\right) q_{k}$. 
Remark 3. The above result can be further improved for some special cases. Let us consider for example $\alpha=(\sqrt{5}-1) / 2 \simeq 0.62$. $\alpha$ is the positive root of the equation $\frac{1}{\alpha}=1+\alpha$ and therefore its partial quotients are all equal to one: $a_{n}=1, \forall n$. Hence for $q_{k}<n \leq q_{k+1}$ the intervals of $\mathcal{Z}^{n}$ have lengths not larger than $2 f_{k}+f_{k+1}(m=1$ and $k^{\prime}=k$ in the proof above). Thus

$$
\underline{R}(x, T, \mathcal{Z}) \geq \lim _{k \rightarrow \infty} \frac{q_{k-1}}{q_{k+1}}=\alpha^{2}=\frac{3-\sqrt{5}}{2},
$$

where for the last identity we have used the fact that in this example the $q_{k}$ 's are the Fibonacci numbers $F_{k}$ defined as $F_{0}=F_{1}=1, F_{k}=F_{k-1}+F_{k-2}, k \geq 2$, and satisfying $F_{k}=\left(\alpha^{-k}-(-\alpha)^{k}\right) / \sqrt{5}$.

We can now formulate the following ${ }^{2}$

Conjecture 1. For all irrational $\alpha$ we have $0 \leq \underline{R}(x, T, \mathcal{Z})<1$, $\mu$-a.e. Moreover $\underline{R}(x, T, \mathcal{Z})>0 \mu$-a.e. if and only if $\alpha$ is of type 1 .

\subsection{Interval Exchange Transformations}

A rotation of the circle is the simplest example of an Interval Exchange Transformation (IET) defined as follows (see [CFS] for details). Let $X=[0,1$ ) as above and let $\xi=\left\{I_{1}, \ldots, I_{r}\right\}, r \geq 2$, be a partition of $X$ into half-open intervals, numbered from left to right, and $\sigma=\left(\sigma_{1}, \ldots, \sigma_{r}\right)$ a permutation of $(1,2, \ldots, r)$. On each half-interval $I_{i}$ the map $T$ acts as a translation $T_{\alpha_{i}} x=x+\alpha_{i}$ in such a way that the intervals get 'exchanged' according to the permutation $\sigma$. The new intervals $T I_{i}=T_{\alpha_{i}} I_{i}=I_{i}^{\prime}$ are thus ordered as $I_{\sigma_{1}}^{\prime}, \ldots, I_{\sigma_{r}}^{\prime}$. In particular the angles $\alpha_{1}, \ldots, \alpha_{r}$ are uniquely determined by the pair $(\xi, \sigma)$. More precisely it holds

$$
\alpha_{i}=\sum_{k<\sigma^{-1}(i)}\left|I_{\sigma_{k}}\right|-\sum_{k<i}\left|I_{k}\right|
$$

EXAMPLE. Let $a, b$ be two real numbers such that $0<a<b<1$ and consider the partition of $[0,1)$ into the half-intervals $I_{1}=[0, a), I_{2}=[a, b)$ and $I_{3}=[b, 1)$, along with the permutation $\sigma=(3,2,1)$. Then we have $I_{\sigma_{1}}^{\prime}=I_{3}^{\prime}=[0,1-b), I_{\sigma_{2}}^{\prime}=I_{2}^{\prime}=[1-b, 1-a)$ and $I_{\sigma_{3}}^{\prime}=I_{1}^{\prime}=[1-a, 1)$. Therefore $T$ is defined as

$$
T x= \begin{cases}x+1-a & \text { if } x \in I_{1} \\ x+1-(a+b) & \text { if } x \in I_{2} \\ x-b & \text { if } x \in I_{3}\end{cases}
$$

Note that $T$ is continuous everywhere but in $a$ and $b$.

It is easy to see that either $T$ has no periodic points or there exists an interval entirely formed by periodic points of the same period. In the former case $T$ is said

\footnotetext{
${ }^{2}$ When this paper was almost finished we received a preprint by M Kupsa entitled Local return rates in Sturmian shifts, whose techniques and results seem promising to prove our Conjecture.
} 
aperiodic. If we denote by $D$ the set of discontinuity points for $T$, that is the set of left-endpoints $d_{i}, i=1, \ldots, r$, of the intervals exchanged by $T$, then aperiodicity is equivalent to the fact that $\cup_{k=0}^{\infty} T^{k} D$ is dense in $[0,1)$. Furthermore, if the full orbits of the points $d_{i}$ are mutually disjoint infinite sets then the dynamical system $(X, T)$ is minimal and uniquely ergodic [CFS]. Finally, an interval exchange transformation cannot be strongly mixing, but it can be weakly mixing [FHZ].

As far as recurrence properties are concerned, very few quantitative result are available. Boshernitzan proved that for an aperiodic exchange of $r$ intervals such that the lengths of all exchanged intervals are algebraic numbers the Hausdorff dimension of any $T$-invariant continuous probability measure $\mu$ on $X$ is bounded below by $1 /(r-1)$ (see [Bo], Corollary 6.9). Therefore, by (2.9) we have that $\nu_{s}(x) \leq 1, \mu$-a.e., for some $s \leq r-1$. On the other hand this result can be interesting only for $r \geq 4$, since for $r=2,3$ it is known that $\mu$ must be the Lebesgue measure and thus $s=1$. In view of the results discussed in the previous subsection we argue that in this case as well recurrence behaviour should be somehow related to arithmetic properties of the lengths of the exchanged intervals or alternatively, by (4.36), to those of the local rotation angles $\alpha_{i}$.

\subsection{AIC for rotations and Interval Exchange Transformations}

We first study the asymptotic behaviour of the AIC for the symbolic sequences generated by the ergodic rotation $(X, T)$, with $X=(0,1], T(x)=x+\alpha(\bmod 1)$ and $\alpha \in \mathbb{R} \backslash \mathscr{Q}$. To study the AIC for IET we will repeatedly apply the same argument.

When studying the behaviour of the AIC, it turns out to be important the knowledge of the continued fraction expansion of both the number $\alpha$ and of the initial condition. We shall use the following estimate for the approximation of a number $\alpha$ by the rationals $\frac{p_{k}}{q_{k}}$, following at once from (4.25):

$$
\frac{1}{q_{k}^{2} \cdot\left(a_{k+1}+2\right)}<\left|\alpha-\frac{p_{k}}{q_{k}}\right|<\frac{1}{q_{k}^{2} \cdot a_{k+1}}
$$

Let $\sigma$ denote the infinite word in $\mathbb{N}^{\mathbb{N}}$ associated to the continued fractions expansion $\left[a_{1}, a_{2}, \ldots\right]$ of the angle of rotation $\alpha$. In the same way let $\left[\xi_{1}, \xi_{2}, \ldots, \xi_{n}, \ldots\right]$ be the continued fraction expansion for a number $x \in(0,1)$ (the initial condition), and denote by $s$ the associated word. We also denote by $\frac{p_{k}}{q_{k}}$ and $\frac{r_{h}}{t_{h}}$, respectively, the rational approximants of $\alpha$ and $x$ :

$$
\frac{p_{k}}{q_{k}}=\left[a_{1}, a_{2}, \ldots, a_{k}\right], \quad \frac{r_{h}}{t_{h}}=\left[\xi_{1}, \xi_{2}, \ldots, \xi_{h}\right] .
$$

Let $\mathcal{Z}$ be the generating partition of $X$ in the intervals $A_{0}=(0,1 / 2]$ and $A_{1}=$ $(1 / 2,1]$. We consider the AIC of the ergodic rotation $(X, T, \mu)$, where $\mu$ is the Lebesgue measure, using this partition.

Theorem 4.10. For all $\alpha \in \mathbb{R} \backslash Q$ and for $\mu$-almost all $x \in X$ we have $A I C(x, n) \approx$ constant. 
Proof. By the estimate (4.38) we have that the knowledge of the first $k+1$ digits (we remark that $n$ digits specify $p_{i}$ and $q_{i}$ for $i=1, \ldots, n$ ) in the continued fractions expansion of $\alpha$ allows us to say that $T^{n}(x)$ is contained in the interval

$$
I_{n}^{k}:=\left[\left(x+n \frac{p_{k}}{q_{k}}-\frac{n}{q_{k}^{2} a_{k+1}}\right)(\bmod 1),\left(x+n \frac{p_{k}}{q_{k}}+\frac{n}{q_{k}^{2} a_{k+1}}\right)(\bmod 1)\right]
$$

The knowledge of the endpoints of this interval yields unambiguously the symbol $\omega_{n}$ in the symbolic orbit of $x$ provided $I_{n}^{k}$ does not contain 0 or $1 / 2$. Let $T_{k}$ be the translation of the angle $\frac{p_{k}}{q_{k}}$ and $\omega^{k}$ be the symbolic orbit of the point $x$ with the map $T_{k}$ on $Z$ : we have $\omega_{n}^{k}=\omega_{n}$ provided $I_{n}^{k}$ does not contain 0 or $1 / 2$. Set

$$
A(k, n):=\left\{x \in X: \omega_{n} \neq \omega_{n}^{k}\right\}
$$

A short calculation yields

$$
\mu(A(k, n)) \leq \frac{4 n}{q_{k}^{2} a_{k+1}}
$$

for all $k$ and $n$. The measure of the set of initial points $x$ that have at least one symbol among $\omega_{1} \ldots \omega_{n}$ different from the corresponding symbols in the word $\omega^{k}$ is then estimated as

$$
\mu\left(\bigcup_{i=1}^{n} A(k, i)\right) \leq \sum_{i=1}^{n} \mu(A(k, i)) \leq \sum_{i=1}^{n} \frac{4 i}{q_{k}^{2} a_{k+1}}=\frac{2 n(n+1)}{q_{k}^{2} a_{k+1}}
$$

Let now $k(n)$ be a sequence of integers such that

$$
\sum_{n=1}^{\infty} \frac{4 n}{q_{k(n)}^{2} a_{k(n)+1}}<\infty
$$

By Borel-Cantelli Lemma we have that for $m$-almost all $x \in X$ there is $n_{0}>0$ such that $x \in X \backslash A(k, n)$ for all $n \geq n_{0}$ and therefore

$$
A I C(x, n)=A I C\left(\omega_{0} \omega_{1} \ldots \omega_{n-1}\right) \leq A I C\left(\omega_{o}^{k(n)} \omega_{1}^{k(n)} \ldots \omega_{n-1}^{k(n)}\right)+\text { const }
$$

that is to specify the first $n$ symbols of the sequence $\omega$ it is enough to specify the first $n$ symbols of the symbolic word $\omega^{k(n)}$ obtained by the orbit of the point $x$ drawn using the periodic map $T_{k(n)}$, defined by $T_{k(n)}(x)=x+\frac{p_{k(n)}}{q_{k(n)}}(\bmod 1)$.

We have now to estimate the information content of the first $n$ symbols of the word $\omega^{k}$. First of all we have that to reconstruct $\omega^{k}$ it is necessary to know $\frac{p_{k}}{q_{k}}$, hence we need to know the first $k$ symbols of the sequence $\sigma$.

Now it remains the uncertainty about the initial condition we use to generate $\omega^{k}$. We repeat the same argument as before. Let $\left[\xi_{1}, \xi_{2}, \ldots, \xi_{n}, \ldots\right]$ be the expansion in continued fractions of the initial condition $x$. If we know only the first $h$ symbols in this expansion, we can approximate $x$ by the rational number $\frac{r_{h}}{t_{h}}$ so that $x+n \frac{p_{k}}{q_{k}}(\bmod 1)$ is contained in the interval

$$
J_{n}^{h}:=\left[\left(\frac{r_{h}}{t_{h}}+n \frac{p_{k}}{q_{k}}-\frac{1}{t_{k}^{2} \xi_{k+1}}\right)(\bmod 1),\left(\frac{r_{h}}{t_{h}}+\frac{p_{k}}{q_{k}}+\frac{1}{t_{k}^{2} \xi_{k+1}}\right)(\bmod 1)\right]
$$


Reasoning as above and choosing $h(n)$ so that

$$
\sum_{n=1}^{\infty} \frac{4}{t_{h(n)}^{2} \xi_{h(n)+1}}<\infty
$$

we conclude that for $m$-almost all $x \in X$ there exists $n_{1}>0$ such that for all $n \geq n_{1}$ it is possible to determine $\omega_{n}^{k}$ just knowing $\left[\xi_{1}, \xi_{2}, \ldots, \xi_{h(n)}\right]$.

From this argument we can conclude that for almost any $x \in X$ we have that there exist integer functions $k(n)$ and $h(n)$ such that for $n$ large enough

$$
\begin{aligned}
A I C(x, n) & \leq A I C\left(\omega_{0}^{k(n)} \omega_{1}^{k(n)} \ldots \omega_{n-1}^{k(n)}\right)+\text { const } \leq \\
& \leq A I C\left(\sigma_{k(n)}\right)+A I C\left(s_{h(n)}\right)+\log _{2} n+\mathrm{const}
\end{aligned}
$$

where $A I C\left(\sigma_{k(n)}\right)$ and $A I C\left(s_{h(n)}\right)$ denote the Algorithmic Information Content of the first $k(n)$ and $h(n)$ symbols of the sequences $\sigma$ and $s$, respectively, whereas the term $\log _{2} n$ is the information needed to reconstruct the $h(n)$-long symbolic orbit of the rational point $\frac{r_{h(n)}}{t_{h(n)}}$ by the map $T_{k(n)}$.

We now study the general problem of estimating $A I C\left(\sigma_{k}\right)$. We have

$$
A I C\left(\sigma_{k}\right) \leq k \log _{2}\left(\max \left\{a_{i}: i=1, \ldots, k\right\}\right)
$$

where we recall that $a_{i}$ are the partial quotients in the expansion of $\alpha$. This estimate comes from the general estimate of the Algorithmic Information Content of a word written on a given alphabet by the length of the word times the logarithm of the number of letters in the alphabet.

To conclude the proof we need the following lemma.

Lemma 4.4. Let $f(k)=\max _{i \leq k} a_{i}$ and $k(n)$ a sequence such that

$$
\sum_{n=1}^{\infty} \frac{4 n}{q_{k(n)}^{2} a_{k(n)+1}}<\infty
$$

Then it is possible to choose $k(n)$ such that $k(n) \log (f(k(n))) \approx$ constant.

Applying this lemma to $A I C\left(\sigma_{k(n)}\right)$ and, with an entirely analogous argument, to $A I C\left(s_{h(n)}\right)$, we obtain the thesis of the theorem by equation (4.41).

Proof of Lemma 4.4. Given $\alpha \in \mathbb{R} \backslash Q$, the best bounds we can give on the denominators $q_{k}$ are

$$
\frac{1}{2 \prod_{i=0}^{k-1} G^{i}(\alpha)}<q_{k}<\frac{1}{\prod_{i=0}^{k-1} G^{i}(\alpha)}
$$

where $G:[0,1] \rightarrow[0,1]$ is the Gauss transformation given by $G(x)=\{1 / x\}$ for $x>0$ and $G(0)=0$. From this we obtain $q_{k} \geq \frac{1}{2} f(k)$ for all $k$. Moreover, as already remarked in Section 4.1, the golden mean $\bar{\alpha}=\frac{\sqrt{5}-1}{2}$ is the number for which the denominators $\bar{q}_{k}$ are the smallest and they are equal to the Fibonacci numbers $F_{k}$. Hence in general, for all irrational $\alpha$ it holds $q_{k} \geq F_{k}$ for all $k$. 
We have thus obtained two different estimates for the denominators $q_{k}$, and the two estimates have the same order if the function $f(k)$ grows exponentially.

When $f(k)=\mathcal{O}(\exp (k))$, we can use the estimate $q_{k} \geq F_{k}$. Hence

$$
\sum_{n=1}^{\infty} \frac{4 n}{q_{k(n)}^{2} a_{k(n)+1}} \leq \sum_{n=1}^{\infty} \frac{4 n}{F_{k(n)}^{2}} \sim \sum_{n=1}^{\infty} \frac{n}{\left(\frac{1}{\bar{\alpha}}\right)^{2 k(n)}}
$$

Hence one can choose $k(n) \sim \log n^{\frac{3}{2}}$ to obtain condition (4.43). Moreover $k \log (f(k))=$ $\mathcal{O}\left(k^{2}\right)$, from which we obtain the thesis of the lemma.

If instead $f(k)$ grows faster than an exponential, then the better estimate for the denominators $q_{k}$ is $q_{k} \geq f(k)$. In this case

$$
\sum_{n=1}^{\infty} \frac{4 n}{q_{k(n)}^{2} a_{k(n)+1}} \leq \sum_{n=1}^{\infty} \frac{4 n}{f^{2}(k(n))}
$$

and condition (4.43) is obtained choosing $f(k(n)) \sim n^{\frac{3}{2}}$. From this is then easy to see that $k(n) \approx$ constant, hence $k(n) \log (f(k(n))) \sim k(n) \log n \approx$ constant.

From the proof of Theorem 4.10 it follows that the uncertainty on the orbits of points $x \in X$ depends on the uncertainty on the initial condition $x$, but also on the uncertainty on the angle $\alpha$, that in principle may need an infinite amount of information to be known.

If $\alpha$ needs a finite amount of information to be known at any accuracy we call it constructive (see Definition 4.2). In this case stronger results can be obtained (Section 4.4).

We now study the more general Interval Exchange Transformations (see Section 4.2).

To study the behaviour of the AIC for orbits of an IET, we use again the continued fraction expansions. Given the left end points $d^{1}=0, d^{2}, \ldots, d^{r}$ of the partition $\xi$ of $X$ associated to the IET, we denote their continued fraction expansions by $\left[\delta_{(i, 1)}, \ldots, \delta_{(i, n)}, \ldots\right]$, for $i=2, \ldots, r$. Let $\frac{p_{(i, k)}}{q_{(i, k)}}=\left[\delta_{(i, 1)}, \ldots, \delta_{(i, k)}\right]$ be the sequence of rational approximants of $d^{i}$, then it holds (see equation (4.38) ) for all $i=2, \ldots, r$

$$
\left|d^{i}-\frac{p_{(i, k)}}{q_{(i, k)}}\right| \leq \frac{1}{q_{(i, k)}^{2} \delta_{(i, k+1)}}
$$

We denote by $i_{0}$ the index $i$ such that the approximation $\frac{1}{q_{(i, k)}^{2} \delta_{(i, k+1)}}$ is the worst.

From the definition of an IET, we have that the angles $\alpha_{j}$ of rotation for the different intervals can be easily obtained from the points $d^{i}$ and the permutation $\sigma$ that defines the transformation. In particular we find that, using the rational numbers $\frac{p_{(i, k)}}{q_{(i, k)}}$ instead of the points $d^{i}$, we have that the angles $\alpha_{j}$ are approximated by rational numbers $\beta_{j}$, such that for all $j=1, \ldots, r$

$$
\left|\alpha_{j}-\beta_{j}\right| \leq \frac{r}{q_{\left(i_{0}, k\right)}^{2} \delta_{\left(i_{0}, k+1\right)}}
$$


Theorem 4.11. For an aperiodic interval exchange transformation $T$ associated to the permutation $\sigma$ and to the partition $\xi$, we have that for $\mu$-almost all $x \in X$ (where $\mu$ denotes the Lebesgue measure) it holds $A I C(x, n) \approx$ constant, where the AIC is considered with respect to the partition $\xi$.

Proof. The proof is analogous to that of Theorem 4.10, Let $x \in X$ be an initial condition for the IET and denote by $\left[\xi_{1}, \ldots, \xi_{n}, \ldots\right]$ its continued fractions expansion. Again let $\frac{r_{h}}{t_{h}}=\left[\xi_{1}, \ldots, \xi_{h}\right]$ be the rational approximants of $x$. From equation (4.38) we have

$$
\left|x-\frac{r_{h}}{t_{h}}\right| \leq \frac{1}{t_{h}^{2} \xi_{h+1}}
$$

It is evident that for $\mu$-almost all $x \in X$ there exist $h$ and $k$ such that the rational numbers $\frac{r_{h}}{t_{h}}$ and $\frac{p_{(i, k)}}{q_{(i, k)}}$, for $i=2, \ldots, r$, are well ordered on the real line. Hence we obtain the value of $\omega_{0}(x)$, that is the first symbol in the symbolic sequence $\omega(x) \in \Omega:=$ $\{1, \ldots, r\}^{\mathbb{N}}$ associated to the IET by the usual method of symbolic representation.

Given that we know $\omega_{0}(x)$, we have that $T(x) \in A_{1}^{(k, h)}$, where

$$
\begin{gathered}
A_{1}^{(k, h)=}\left[\frac{r_{h}}{t_{h}}+\beta_{\omega_{0}(x)}-\epsilon_{1}(h, k), \frac{r_{h}}{t_{h}}+\beta_{\omega_{0}(x)}+\epsilon_{1}(h, k)\right] \\
\epsilon_{1}(h, k)=\frac{1}{t_{h}^{2} \xi_{h+1}}+\frac{r}{q_{\left(i_{0}, k\right)}^{2} \delta_{\left(i_{0}, k+1\right)}}
\end{gathered}
$$

Hence $\omega_{1}(x)$ is well defined if the set $A_{1}^{(k, h)}$ does not intersect the intervals

$$
B(i, k)=\left[d^{i}-\frac{1}{q_{\left(i_{0}, k\right)}^{2} \delta_{\left(i_{0}, k+1\right)}}, \quad d^{i}+\frac{1}{q_{\left(i_{0}, k\right)}^{2} \delta_{\left(i_{0}, k+1\right)}}\right]
$$

for all $i=1, \ldots, r$. Then if we define

$$
P_{1}^{(k, h)}=\left\{x \in X / A_{1}^{(k, h)} \cap B(i, k) \neq \emptyset \text { for some } i\right\}
$$

we have easily that

$$
\mu\left(P_{1}^{(k, h)}\right) \leq r\left(\frac{2}{t_{h}^{2} \xi_{h+1}}+\frac{2(r+1)}{q_{\left(i_{0}, k\right)}^{2} \delta_{\left(i_{0}, k+1\right)}}\right)
$$

Let us assume now that we know $\omega_{0}(x)$ and $\omega_{1}(x)$. We can then say that $T^{2}(x) \in$ $A_{2}^{(k, h)}$, where now

$$
\begin{gathered}
A_{2}^{(k, h)}=\left[\frac{r_{h}}{t_{h}}+\beta_{\omega_{0}(x)}+\beta_{\omega_{1}(x)}-\epsilon_{2}(h, k), \frac{r_{h}}{t_{h}}+\beta_{\omega_{0}(x)}+\beta_{\omega_{1}(x)}+\epsilon_{2}(h, k)\right] \\
\epsilon_{2}(h, k)=\frac{1}{t_{h}^{2} \xi_{h+1}}+\frac{2 r}{q_{\left(i_{0}, k\right)}^{2} \delta_{\left(i_{0}, k+1\right)}}
\end{gathered}
$$


Again $\omega_{2}(x)$ is well defined if the set $A_{2}^{(k, h)}$ does not intersect the intervals $B(i, k)$. Then we define

$$
P_{2}^{(k, h)}=\left\{x \in X / A_{2}^{(k, h)} \cap B(i, k) \neq \emptyset \text { for some } i\right\}
$$

and obtain

$$
\mu\left(P_{2}^{(k, h)}\right) \leq r\left(\frac{2}{t_{h}^{2} \xi_{h+1}}+\frac{2(2 r+1)}{q_{\left(i_{0}, k\right)}^{2} \delta_{\left(i_{0}, k+1\right)}}\right)
$$

Iterating this argument, we can conclude that for a given $n$, the measure of the set of initial conditions $x$ for which the knowledge of $\frac{r_{h}}{t_{h}}$ and $\frac{p_{(i, k)}}{q_{(i, k)}}$, for $i=2, \ldots, r$, is not enough to construct $\left(\omega_{0}(x), \ldots, \omega_{n-1}(x)\right)$, is estimated from above by

$$
\mu\left(\bigcup_{j=1}^{n} P_{j}^{(k, h)}\right) \leq \sum_{j=1}^{n} r\left(\frac{2}{t_{h}^{2} \xi_{h+1}}+\frac{2(j r+1)}{q_{\left(i_{0}, k\right)}^{2} \delta_{\left(i_{0}, k+1\right)}}\right)
$$

At this point the conclusion of the theorem follows as for Theorem 4.10. From the Borel-Cantelli Lemma, choosing sequences $k(n)$ and $h(n)$ such that

$$
\sum_{n=1}^{\infty} r\left(\frac{2}{t_{h(n)}^{2} \xi_{h(n)+1}}+\frac{2(n r+1)}{q_{\left(i_{0}, k(n)\right)}^{2} \delta_{\left(i_{0}, k(n)+1\right)}}\right)<\infty
$$

we have that for $\mu$-almost all initial conditions $x$

$$
\begin{aligned}
A I C(x, n, Z) \leq & \text { const }+\log _{2} n+A I C\left(\xi_{1}, \ldots, \xi_{h(n)}\right)+ \\
& +\sum_{i=2}^{r} \operatorname{AIC}\left(\delta_{1}^{i}, \ldots, \delta_{k(n)}^{i}\right)
\end{aligned}
$$

where the constant contains also the information necessary to transmit the permutation $\sigma$ to which the IET is associated, and the term $\log _{2} n$ accounts for the information necessary to transmit the number $n$ of iterations that have to be performed using the rational numbers $\frac{r_{h}}{t_{h}}$ and $\frac{p_{(i, k)}}{q_{(i, k)}}$, for $i=2, \ldots, r$.

Simple application of equation (4.42) and Lemma 4.4now conclude the theorem.

As said before for the irrational rotations, if all the coefficients of the IET are constructive numbers, stronger results are possible. This is shown in the next Section.

Remark 4. Theorems 4.10 and 4.11 remain unchanged if we estimate the AIC with respect to any finite measurable partition whose sets are finite union of intervals. In the proofs we just need to add the information coming from the end points of the intervals of the partition, but Lemma 4.4 applies again for these points.

\subsection{Constructive piecewise isometries}

In this section we use Theorem 2.1 and some result from $\mathrm{Ga}$ about the relation between information and initial condition sensitivity in the 0-entropy case to estimate the information content of orbits coming from certain constructive piecewise isometries. 
Piecewise isometries can be somehow sensitive to initial conditions because of their discontinuities. The orbit of two nearby starting points can be separated when the points falls on the different sides of a discontinuity.

Theorem 2.1 gives a result about quantitative recurrence rates of measure preserving maps in a neighbourhood of some target points. The theorem allows to estimate the distance of a typical orbit from the discontinuity points and then to estimate this kind of sensitivity.

Now we define the class of maps which will be studied. A real number is constructive if it can be approximated at any accuracy by an algorithm.

Definition 4.2 (Constructive numbers). A number $z \in \mathbf{R}$ is said to be constructive if there is an algorithm $A_{z}(n): \mathbf{N} \rightarrow \mathbf{Q}$ such that $A_{z}(n)=q$ implies $|q-z|<2^{-n}$.

This definition was already given by Turing $([\mathrm{T}])$ and the study of the computable properties of real numbers was one of the motivations he had in mind when introducing his famous computing machines.

Definition 4.3. A constructive piecevise isometry on the interval is a piecewise isometry $T$ with the following properties: it is Lebesgue measure preserving and both the discontinuity points $y_{1}, \ldots, y_{n}$ and the values $T\left(y_{1}\right), \ldots, T\left(y_{n}\right)$ of $T$ at the discontinuity points are constructive numbers.

Rotations and interval exchanges whose angles are constructive are included in the previous definition.

If a map satisfies the above definition, for each constructive point $x \in[0,1]$ such that $x \notin\left\{y_{1}, \ldots, y_{n}\right\}$ the value of $T(x)$ can be calculated up to any given accuracy by an algorithm. This implies the following lemma

Lemma 4.5. Let $T$ be a constructive piecewise isometry then there is a constructive number $r \in[0,1]$, such that for each rational $q$, the orbit of the point $x=q+r \bmod (1)$ $\in[0,1]$ can be followed at any accuracy by an algorithm, in the following sense:

there is an algorithm (a total recursive function) $A: \mathbf{Q} \times \mathbf{N} \times \mathbf{Q} \rightarrow \Sigma$ such that $\forall k \in \mathbf{N}, q \in \mathbf{Q}, \epsilon \in \mathbf{Q} d\left(T^{k}(x),(A(q, k, \epsilon)+r) \bmod (1)\right)<\epsilon$.

Proof. For the proof we refer to the results that are in Ga Section 3. Let us consider the following set $B=\left\{x \in[0,1], \exists i, j\right.$ s.t. $\left.T^{i}(x)=y_{j}\right\}$ and $X^{\prime}=[0,1]-B$. Let us consider a constructive number $r$ such that $r$ is incommensurable with all $y_{i}$ and $T\left(y_{i}\right)$. Let us consider the standard interpretation (Ga Def. 3) on [0,1] $I$ given by $I(s)=\sum 2^{-s_{i}}$ where $s=s_{1} s_{2} \ldots s_{n} \in \Sigma$. Consider the computable structure $\mathcal{I}$ containing $I$. Now let us consider $r$ and $I_{r}$ given by

$$
I_{r}(s)=I(s)+r(\bmod 1)
$$

then $I_{r}$ is a computable interpretation and is contained in $I . I_{r}(\Sigma)$ is contained in $X^{\prime}$ and let $\mathcal{I}_{r}$ be the computable structure containing $I_{r}$.

Now the system $\left(X^{\prime}, \mathcal{I}_{r}, T\right)$ is constructive (for [Ga] Definition 9 ) and $T$ is continuous over $X^{\prime}$ and the assumptions of [Ga Lemma 10 are satisfied. Then [Ga] Lemma 10 holds for all $x \in X^{\prime}$ and then for all $x \in I_{r}(\Sigma)$, easily implying the statement. 
Let us now consider a constructive piecewise isometry as in definition above. Let $y_{1}, \ldots, y_{l}$ be the discontinuity points. Let us consider two nearby starting points. As it was said before, during the iterations of the map the orbits can be separated by the discontinuity, and the map is in a certain sense sensitive to initial conditions. This sensitivity will be estimated by the use of Theorem 2.1. Now let us consider a partition $\alpha$ and suppose that $\alpha$ is finite, and each set of $\alpha$ is made of a finite union of intervals whose end points are constructive numbers. Let us consider $A I C(x, \alpha, n)$, the information content of the orbit of $x$ with respect to the partition.

Theorem 4.12. Let $([0,1], T)$ be an IET with flips as in Def. [4.3, then for Lebesgue a.e. $x \in X$

$$
\varlimsup_{n \rightarrow \infty} \frac{A I C(x, \alpha, n)}{\log n} \leq 2 .
$$

Proof. Refining the partition, the information content increases, then we can suppose that in the partition $\alpha=\left\{\left[\alpha_{1}, \alpha_{2}\right),\left[\alpha_{2}, \alpha_{3}\right), \ldots,\left[\alpha_{q-1}, \alpha_{q}\right]\right\}$ each set is smaller than the smallest discontinuity of $T$.

Let

$$
B(n, x, \alpha)=\left\{y \in X \text { s.t. } \forall i \leq n, T^{i}(y) \in\left[\alpha_{j}, \alpha_{j+1}\right) \Leftrightarrow T^{i}(x) \in\left[\alpha_{j}, \alpha_{j+1}\right)\right\}
$$

be the set of points having the same symbolic orbit as $x$ for $n$ steps.

Let us consider two nearest points $x, y$, starting from the same set of $\alpha$. Since $T$ is a piecewise isometry $x$ and $y$ continue to have the same symbolic orbit until

1) they are separated by a discontinuity, or

2) they go to different sets of $\alpha$, because a point $\alpha_{i}$ separating different sets of $\alpha$ comes between their images $\left(\exists j, i\right.$ such that $T^{j}(x) \leq \alpha_{i} \leq T^{j}(y)$ or $T^{j}(x) \geq \alpha_{i} \geq$ $\left.T^{j}(y)\right)$.

Moreover, this implies $B(n, x, \alpha)$ is an interval for each $n$.

Since $T$ is a piecewise isometry the two above cases does not happen for $n$ steps if the orbit of $x$ remains far away enough from the discontinuity and separation points, i.e. if $\forall i \leq n \min _{j}\left(\min \left(d\left(T^{n}(x), y_{j}\right), d\left(T^{n}(x), \alpha_{j}\right)\right)\right)>d(x, y)$. Now we apply Theorem 2.1 to $T$ with the Lebesgue measure (which has local dimension 1 for each point), where the $x_{i}$ in the statement are the union of discontinuity points of $T$ and the separation points of $\alpha\left(\left\{x_{i}\right\}=\left\{y_{i}\right\} \cup\left\{\alpha_{i}\right\}\right)$. By this theorem for a.e. point $x$ the length of the interval $B(n, x, \alpha)$ decreases eventually slower than $\frac{C}{n^{p}}$ for some $C$ and each $p>1$.

Now let us consider $r$ found in Lemma 4.5. We remark that in an interval with length $\ell$ there is at least a rational $r$ of the kind $r=0 . s_{1} s_{2} \ldots s_{v}$ with $v \leq-\log \ell+1$.

By the estimation above about the size of $B(n, x, \alpha)$, for a.e. $x$, for each $n$ there is $\tilde{x} \in B(n, x, \alpha)$ such that $\tilde{x}=0 . s_{1} s_{2} \ldots s_{v}+r(\bmod 1)$ is such that $v<p \log n+C$, $\forall p>1$ and the point $\tilde{x}$ will have the same symbolic orbit as $x$.

It is possible to construct a program giving the symbolic orbit of $x$ with the information contained in the map $T$, in the string $\left[s_{1} s_{2} \ldots s_{v}\right]$ and in the number of steps $n$.

The program giving the symbolic orbit of $x$ will contain a string $\left[s_{1} s_{2} \ldots s_{v}\right](p \log n+$ $C$ bits, for $p$ near to 1 as we want), the information that is necessary to know $T$ and $p$ (a constant). 
A program which outputs the symbolic sequence associated to the orbit of $x$ will run as follows:

by Lemma 4.5 it will follow the orbit of $\tilde{x}$ with an accuracy $1 / k$ obtaining points $\tilde{x}_{1}(\epsilon), \ldots, \tilde{x}_{n}(\epsilon)$ following the orbit of $\tilde{x}$ at a distance less than $1 / k$. The procedure is repeated increasing $k$ until $\min _{j, i}\left(d\left(\tilde{x}_{i}(\epsilon), \alpha_{j}\right)\right)>2 / k$ (this assures that the sequence $\tilde{x}_{1}(\epsilon), \ldots, \tilde{x}_{n}(\epsilon)$ has the same symbolic orbit as $x$ with respect to the partition $\alpha$.

Once having the sequence $\tilde{x}_{1}(\epsilon), \ldots, \tilde{x}_{n}(\epsilon)$ the symbolic orbit with respect to $\alpha$ is calculated and this is the output of the computation. By what is said above the length of the program is then less or equal to $2 \log n+C$.

\section{References}

[AB] P AlEssandri, V Berthì, Three distances theorem and combinatorics on words, L'Enseignement Mathématique 44 (1998), 103-132

[ACS] V Afraimovich, J R Chazottes, B Saussol, Pointwise dimensions for Poincaré recurrences associated with maps and special flows, Disc. Cont. Dyn. Syst. - A 9 (2003), 263-280

[BS] L Barreira, B SAussol, Hausdorff dimension of measures via Poincaré recurrence, Commun. Math. Phys. 219 (2001), 443-463

[BCF] V Berthè, N Chekhova, S Ferenczi, Covering numbers: arithmetics and dynamics for rotations and interval exchanges, Journal d'Analyse Mathématique 79 (1999), 1-31

[B] C Bonanno, Application of Information Measures to Chaotic Dynamical Systems, Ph.D. thesis, University of Pisa, 2003

[BoGa] C Bonanno, S Galatolo, Orbit complexity for the Manneville maps, in preparation

[Bo] M D Boshernitzan, Quantitative recurrence results, Invent. Math. 113 (1993), 617-631

[Br] A Brudno, Entropy and the complexity of trajectories of dynamical systems, Russ. Math. Surv. 2 (1983), 127-151 (English transl.)

[CFS] I P Cornfeld, S V Fomin And Ya G Sinai, Ergodic Theory, Springer Verlag, 1982

[Fa] K J FAlconer, The Geometry of Fractal Sets, Princeton University Press, 1981

[FHZ] S Ferenczi, C Holton, L Zamboni, Structure of three-interval exchange transformations III: ergodic and spectral properties, preprint (2000) 
[Fu H Furstenberg, Recurrence in Ergodic Theory and Combinatorial Number Theory, Cambridge University Press, 1990

[Ga] S Galatolo, Complexity, initial condition sensitivity, dimension and weak chaos in dynamical systems, Nonlinearity 16 (2003), 1219-1238

[HSV] M Hirata, B Saussol, S Vaienti, Statistics of return times: a general framework and new applications, Commun. Math. Phys. 206 (1999), 33-55

[KH] A Katok, B Hasselblatt, Introduction to the Modern Theory of Dynamical Systems, Cambridge University Press, 1995

[Ka] M KAC, On the notion of recurrence in discrete stochastic processes, Bull. Amer. Math. Soc. 53 (1947), 1002-1010

[OW] D S Ornstein, B Weiss, Entropy and data compression schemes, IEEE Trans. Inf. Th. 39 (1993), 78-83

[Pe] K Petersen, Ergodic Theory, Cambridge University Press, 1983

[Pr] T Prellberg, Maps of Intervals with Indifferent Fixed Points: Thermodynamic Formalism and Phase Transitions, Ph.D. thesis, Virginia Polytechnic Institute and State University, 1991

[RS] A M Rocketr, P Szüsz, Continued Fractions, World Scientific, 1992

[STV] B Saussol, S Troubetzkoy, S Vaienti, Recurrence, dimensions and Lyapunov exponents, J. Stat. Phys. 106 (2002), 623-634

[T] A Turing, On computable numbers, with an application to the "Entscheidungsproblem", Proc. Lond. Math. Soc. 42 (1936), 230-265

[W] H S White, Algorithmic complexity of points in dynamical systems, Erg. Th. Dyn. Syst. 13 (1993), 807-830

[Y] L-S Young, Dimensions, entropy and Lyapunov exponents, Erg. Th. Dyn. Syst. 2 (1982), 109-124 